\title{
Technology Enhanced Interaction Framework
}

\author{
Kewalin Angkananon \\ ECS \\ University of Southampton \\ Southampton, UK \\ ka3e10@ecs.soton.ac.uk
}

\author{
Mike Wald, Lester Gilbert \\ ECS \\ University of Southampton \\ Southampton, UK \\ mw@ecs.soton.ac.uk,1g3@ecs.soton.ac.uk
}

\begin{abstract}
This paper focuses on the development of a general interaction framework to help design technology to support communication between people and improve interactions between people, technology and objects, particularly in complex situations. A review of existing interaction frameworks shows that none of them help technology designers and developers to consider all of the possible interactions that occur at the same time and in the same place. The main and sub-components of the framework are described and explained and examples are given for each type of interaction. Work is now in progress to provide designers with an easy to use tool that helps them apply the framework to create technology solutions to complex communication and interaction problems and situations.
\end{abstract}

Keywords- Interaction, framework, technology, design

\section{INTRODUCTION}

As information and communication technology has become more important in society, many researchers have been concerned with how to use technology to support communication between people and improve interactions between people, technology and objects $[1 ; 5 ; 6 ; 14 ; 17 ; 19$; 20]. There has, however, been no framework that has helped technology designers and developers to consider all of the possible interactions that occur at the same time and in the same place although there have been projects concerned with how to use technology to support some of these interactions. For example, artefact-mediated-communication has been used to support cooperative work $[4 ; 5 ; 6 ; 20]$, a mobile digital guidebook has been used to enhance visitors' interaction with physical objects in museums $[11 ; 19]$ and mobile devices have been used as mediators for the interaction with a physical object using QR codes, RFID tags and NFC tags [2; 17].

Many publications and projects in human computer interaction (HCI) focus on using technologies as a tool to enhance experiences: in the same place but at a different time (e.g. using systems for supporting group learning such as notice boards, questions and answers, electronic debates and collaborative learning [15]); in a different place but at the same time (e.g. using a Synchronous Communication Tool such as video conferencing, instant messaging and online chats to interact with learners to improve their communication with the instructor [21]); and in a different place at a different time (e.g. using blended learning, students can access e-learning in order to learn in a different place at a different time [12]).

This paper focuses on the development of a general interaction framework adapted from and extending the work of Dix [7] and Gaines [10] to help design technology to support communication between people and improve interactions between people, technology and objects, particularly in complex situations. The paper is structured as follows. Section II reviews previous research on interaction frameworks, section III explains the Technology Enhanced Interaction Framework and section IV describes the future work taking place to enable the framework to help developers design technology to enhance face-to-face interaction in the same time and the same place.

\section{REVIEW OF INTERACTION FRAMEWORKS}

Table 1 summarises a review of interaction frameworks and shows that many frameworks focus on people to people communication in the same time and at the same place but not using technology to enhance communication. Some frameworks address many interactions between humans and computers $[6 ; 19]$.

A framework for Computer Supported Cooperative Work [7] seems to address some of the possible interactions but it misses out some important interactions in the same time and at the same place situations such as people using technology to interact with real objects.

In Dix's framework, the participants communicate with other participants in what is called "direct communication". Furthermore, the participants also interact with artefacts (manmade technology tools) by "controlling" or "acting". Sometimes an artefact is shared between the participants; in this case, the artefact is not only the subject of communication but can become a medium of communication, called "feedthrough". In communication about work and the artefacts of work, various means are used to refer to particular artefacts, and Dix terms this "deixis", as shown in Fig. 1.

No current framework addresses all of the interactions identified in Table 1. The Technology Enhanced Interaction Framework of this paper addresses this, as explained in the next section. 
TABLE 1 Summarising a review of frameworks of interactions

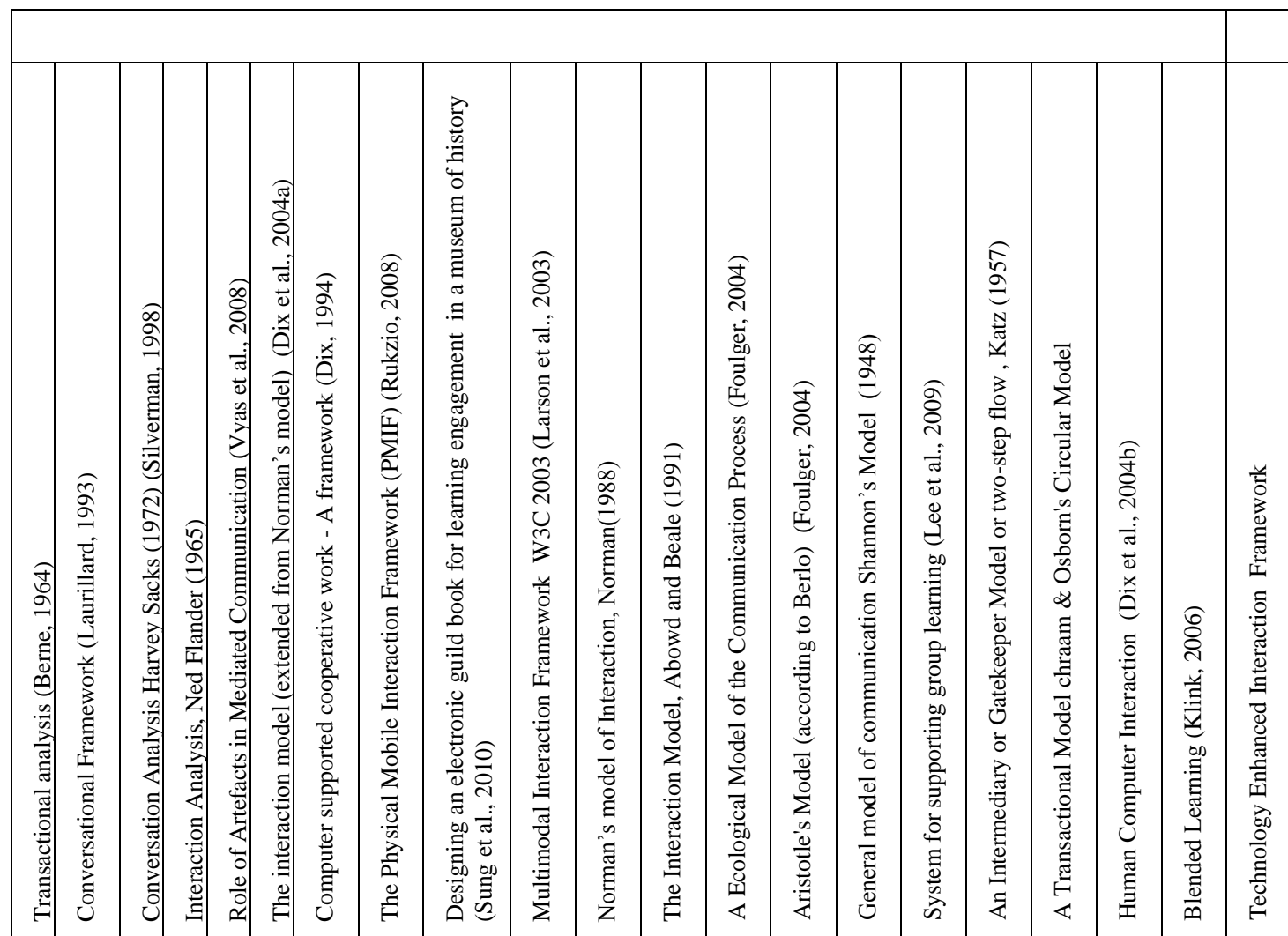

\begin{tabular}{|c|c|c|c|c|c|c|c|c|c|c|c|c|c|c|c|c|c|c|c|c|c|}
\hline \multirow{2}{*}{\multicolumn{22}{|c|}{ Direct Communication }} \\
\hline & & & & & & & & & & & & & & & & & & & & & \\
\hline People-People & $\checkmark$ & $\checkmark$ & $\checkmark$ & $\checkmark$ & & & $\checkmark$ & & & & & & $\checkmark$ & $\checkmark$ & $\checkmark$ & & $\checkmark$ & $\checkmark$ & & $\checkmark$ & $\checkmark$ \\
\hline \multicolumn{22}{|l|}{ Interactions } \\
\hline People-Technology & & & & & & $\checkmark$ & & $\checkmark$ & $\checkmark$ & $\checkmark$ & $\checkmark$ & $\checkmark$ & & & & $\checkmark$ & & & $\checkmark$ & $\checkmark$ & $\checkmark$ \\
\hline People-Object & & & & & & & & & & & & & & & & & & & & & $\checkmark$ \\
\hline $\begin{array}{l}\text { People-technology- } \\
\text { people }\end{array}$ & & & & & $\checkmark$ & & $\checkmark$ & $\checkmark$ & $\checkmark$ & & & & & & & $\checkmark$ & & & $\checkmark$ & $\checkmark$ & $\checkmark$ \\
\hline $\begin{array}{l}\text { People-technology- } \\
\text { object }\end{array}$ & & & & & & & & & $\checkmark$ & & & & & & & & & & & & $\checkmark$ \\
\hline \multicolumn{22}{|l|}{ Role of interaction } \\
\hline Presenter-Audience & & & & & & & & & & & & & & & & & & & & & $\checkmark$ \\
\hline Sender-Receiver & & & & & & & & & & & & & & & $\checkmark$ & & $\checkmark$ & $\checkmark$ & & & $\checkmark$ \\
\hline Teacher-Student & & $\checkmark$ & & $\checkmark$ & & & & & & & & & & & & & & & & & $\checkmark$ \\
\hline Consumer-creator & & & & & & & & & & & & & $\checkmark$ & & & & & & & & $\checkmark$ \\
\hline Speaker-Audience & & & & & & & & & $\checkmark$ & & & & & $\checkmark$ & & & & & & & $\checkmark$ \\
\hline User-system & & & & & $\checkmark$ & $\checkmark$ & & & & $\checkmark$ & $\checkmark$ & $\checkmark$ & & & & $\checkmark$ & & & $\checkmark$ & $\checkmark$ & $\checkmark$ \\
\hline Peer-peer & & & & & & & $\checkmark$ & $\checkmark$ & & & & & & & & $\checkmark$ & & & & & $\sqrt{ }$ \\
\hline No role & $\checkmark$ & & $\checkmark$ & & & & & $\checkmark$ & & & & & & & & & & & & & $\checkmark$ \\
\hline \multicolumn{22}{|l|}{ Space/Time } \\
\hline Same place/same time & $\checkmark$ & $\checkmark$ & $\checkmark$ & $\checkmark$ & $\checkmark$ & & $\checkmark$ & $\checkmark$ & $\checkmark$ & & $\checkmark$ & $\checkmark$ & $\checkmark$ & $\checkmark$ & $\checkmark$ & & $\checkmark$ & $\checkmark$ & & $\checkmark$ & $\checkmark$ \\
\hline $\begin{array}{l}\text { Same place/different } \\
\text { time }\end{array}$ & & & & & & $\checkmark$ & & & & $\checkmark$ & & & & & & $\checkmark$ & & & & $\checkmark$ & $\checkmark$ \\
\hline $\begin{array}{l}\text { Same time/different } \\
\text { place }\end{array}$ & & & & & & $\checkmark$ & & & & $\checkmark$ & & & & & & & & & & $\checkmark$ & $\checkmark$ \\
\hline $\begin{array}{l}\text { Different time/different } \\
\text { place }\end{array}$ & & & & & & $\checkmark$ & & & & $\checkmark$ & & & & & & & & & & $\checkmark$ & $\checkmark$ \\
\hline \multicolumn{22}{|l|}{ Technology enhancement } \\
\hline Using technologies & & & & & $\checkmark$ & $\checkmark$ & & $\checkmark$ & $\checkmark$ & $\checkmark$ & & & & & & & & & $\checkmark$ & $\checkmark$ & $\checkmark$ \\
\hline Without technology & $\checkmark$ & $\checkmark$ & $\checkmark$ & $\checkmark$ & & & $\checkmark$ & & & & $\checkmark$ & $\checkmark$ & $\checkmark$ & $\checkmark$ & $\checkmark$ & & $\checkmark$ & $\checkmark$ & & & $\checkmark$ \\
\hline \multicolumn{22}{|l|}{ Accessibility } \\
\hline Consider accessibility & & & & & & & & & & & & & & & & & & & & & $\checkmark$ \\
\hline
\end{tabular}




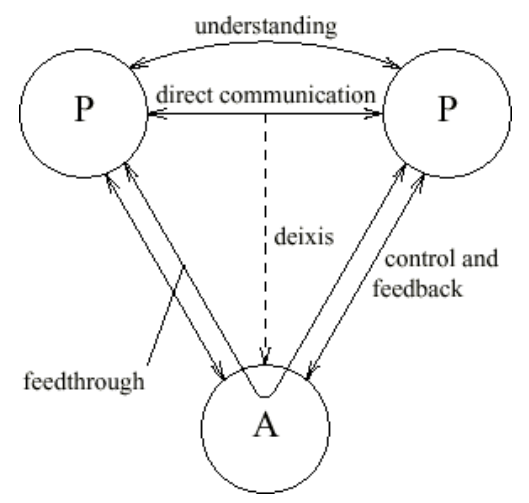

Figure 1 Computer Supported Cooperative Work - A framework (Dix, 1994)

\section{THE TECHNOLOGY ENHANCED INTERACTION FRAMEWORK}

The Technology Enhanced Interaction Framework supports the design of technology enhanced interactions by developers and designers.

\section{A. Terminology}

- Communication is the process of passing information from one person to another [3].

- Technology is a tool that helps people achieve their purpose.

- People means anyone involved in direct communication or interaction with an object, technology, or other people.

- Object is anything that is not a technology or a person involved in communication or interaction.

- Interactions can be between people and objects (P-O) or people and technology (P-T). People can also use technology to mediate interaction with people (P-T-P) or objects (P-T-O).

\section{B. Main components}

There are seven main components in the Technology Enhanced Interaction Framework as shown in Table 2. People can have roles, abilities, and disabilities. The components "Object" and "Technology" are used in order to extend Dix's framework to show any type of interaction. Objects are defined as having three sub-components: dimensions, properties, and content. Technology has a cost and can be electronic or nonelectronic, online or off-line, and mobile or non-mobile. Furthermore, it may or may not have stored content and may additionally have an interface and be an application or provide a service. Interactions and communication are classified into three groups:
1) Direct Communication:

a) People to People (P-P) - People in one way or two way communication with people.

2) Direct Interaction:

a) People to Technology (P-T) - People can control technology and may also be able to use it to store or retrieve information.

b) People to Objects ( $\mathrm{P}-\mathrm{O})$ - People can control objects and retrieve information from objects.

3) Technology Mediated Interaction:

a) People to Technology to People (P-T-P) -Technology can mediate communication between people.

b) People to Technology to Objects (P-T-O) - People can control objects with Technology and may also be enabled to use objects to store and retrieve information.

Time and Place can be divided into four categories [8] same time and same place, different time but same place, same time but different place, and different place and different time.

Context can include factors and constraints such as location, signal quality, background noise, and weather conditions.

The role played by the interactions and communication may be classified into one of six interaction layers, adapted from Gaines [10] as follows:

- Cultural layer includes countries, tradition, language, and gesture.

- Intentionality layer involves understanding, purpose and benefit.

- Knowledge layer involves facts, concepts, and principle [16].

- Action layer involves actions and procedures [16].

- Expression layer describes how actions are carried out (e.g. correctly or with errors).

- Physical layer is the lowest layer at which people interact with the physical world.

For example, pressing of the letter " $h$ " on the keyboard when typing "hello" as a greeting when sending a text message can be thought of as:

- Cultural layer: "hello" is a normal greeting used in the culture.

- Intentionality layer: the intent is a greeting.

- Knowledge layer: how to spell the word "hello".

- Action layer: pressing key ' $h$ '.

- Expression layer: pressing the correct key and not hitting neighbouring keys.

- Physical layer: the button is depressed and so sends the electronic code for the letter to the application. 
TABLE 2 Main Component of Technology Enhanced Interaction Framework

\begin{tabular}{|c|c|c|}
\hline \multirow{2}{*}{ Main Component } & \multicolumn{2}{|c|}{ Main Component of Technology Enhanced Interaction Framework } \\
\hline & Sub-component & Example \\
\hline \multirow{2}{*}{ People } & Role & $\begin{array}{l}\text { speaker - audience (e.g. teacher - student ; owner - visitor), } \\
\text { peer-peer }\end{array}$ \\
\hline & Ability/disability & $\begin{array}{l}\text { physical disability, sensory disability, language, culture, } \\
\text { communication, Information Technology (IT) }\end{array}$ \\
\hline \multirow{3}{*}{ Objects } & Dimension & 2 dimensional (2D), 3 dimensional (3D) \\
\hline & Property & colour, shape, size \\
\hline & Content & $\begin{array}{l}\text { human readable (text, pictures, audio, video), } \\
\text { machine readable (QR codes, AR tag, barcodes, RFID tag, NFC) }\end{array}$ \\
\hline \multirow{5}{*}{$\begin{array}{l}\text { Interactions and } \\
\text { communication }\end{array}$} & People-People(P-P) & $\begin{array}{l}\text { verbal communication (speak, listen, ask, answer), } \\
\text { non-verbal (lip-read, smile, touch, sign, gesture, nod), } \\
\text { deixis (refer) }\end{array}$ \\
\hline & People-Objects(P-O) & $\begin{array}{l}\text { control (touch, hold, move), } \\
\text { information retrieval: } \\
\text { - knowledge (look, listen, read, remember colour, shape, size) } \\
\text { - meaning objects (understand) }\end{array}$ \\
\hline & People-Technology(P-T) & $\begin{array}{l}\text { control (hold, move, use compass, type, scan, take photo, press, swipe), } \\
\text { information transmission and storage (send information, save, store, } \\
\text { search online or offline document, retrieve) }\end{array}$ \\
\hline & People-Technology-People(P-T-P) & $\begin{array}{l}\text { control (send sms, mms, email, show information, chat), } \\
\text { information transmission and storage (send information, save, store, } \\
\text { search, retrieve) }\end{array}$ \\
\hline & People-Technology-Objects(P-T-O) & $\begin{array}{l}\text { control (point, move, hold, scan QR codes, scan AR tag, use camera, } \\
\text { use compass), } \\
\text { information transmission and storage (send information, save, store, } \\
\text { search, retrieve) }\end{array}$ \\
\hline \multirow{5}{*}{ Technology } & $\begin{array}{l}\text { Electronic } \\
\text { (store information in technology) }\end{array}$ & online, offline, content, non-content, mobile, non-mobile \\
\hline & $\begin{array}{l}\text { Non-electronic } \\
\text { (store information in objects) }\end{array}$ & content, non-content, mobile, non-mobile \\
\hline & Interface & website, mobile website \\
\hline & Application or service & mobile website, mobile application \\
\hline & Cost & hardware, software, staff \\
\hline \multirow{2}{*}{ Time/Place } & Place & same place (SP), different place (DP) \\
\hline & Time & same time (ST), different time (DT) \\
\hline \multirow{4}{*}{ Context } & Location & indoor/outdoor \\
\hline & Weather condition & rainy, cloudy, sunny, windy, hot, cold, dry, wet \\
\hline & Signal type and quality & broadband, GPS, 3G \\
\hline & Background Noise & background music, crowded situation \\
\hline \multirow{6}{*}{ Interaction layer } & Culture & gesture, language \\
\hline & Intentionality & understand, purpose, benefit \\
\hline & Knowledge & Facts, concepts, principle \\
\hline & Action & touch, move, type, swipe \\
\hline & Expression & whether action is correct, accurate, prompt \\
\hline & Physical & colour, size, shape \\
\hline
\end{tabular}




\section{Architecture of the Technology Enhanced Interaction Framework}

The overall architecture of the Technology Enhanced Interaction Framework involves people, technology and objects (Fig. 2). The general framework covers the use of any technology, which may or may not be electronic; the main difference is that electronic technology can store information. The Technology Enhanced Interaction Framework extends Dix's framework [7] for computer supported cooperative work (Fig. 1) to include interaction with objects.

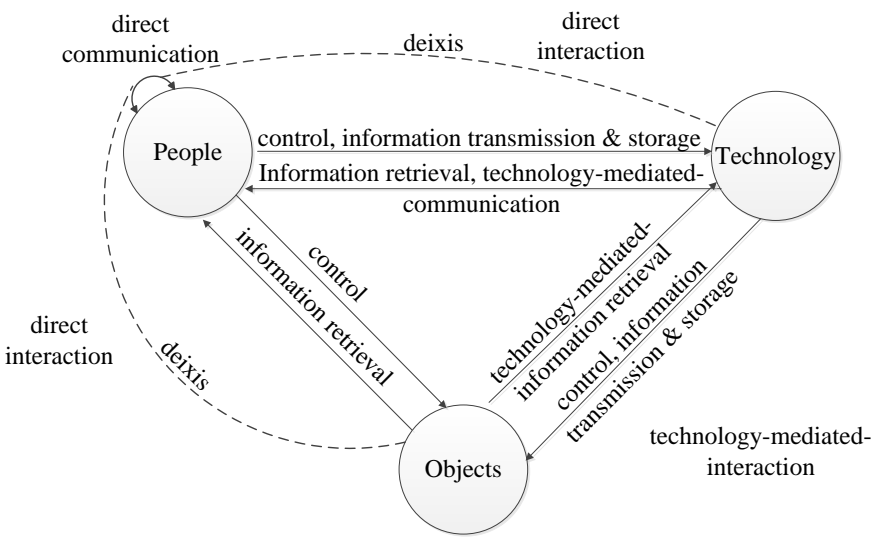

Figure 2 The Technology Enhanced Interaction Framework extended from Dix [7]

The Technology Enhanced Interaction Framework involves interactions from people to people $(\mathrm{P}-\mathrm{P})$, people to objects (P-O), people to technology (P-T), people to technology to people (P-T-P) and people to technology to objects (P-T-O) as follows:

\section{1) Direct communication}

a) People to People (P-P)

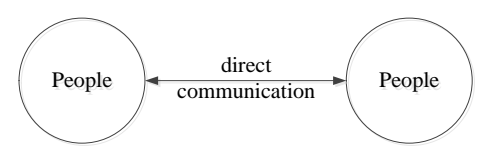

Figure 3 Direct communication between people

The communication between people and people (Fig. 3) is a complex subject [7]. Bern [1] identified three roles of parent, adult, and child in his theory of Transactional Analysis. The conversational framework developed by Laurillard [14] describes how the roles of teachers and students interact in the learning and teaching process. Apart from a role, people have abilities or disabilities which can affect their use of technology or understanding of language and which can lead to communication breakdown. In direct communication people may refer to particular objects and technology - this is known as deixis [7].

An example of direct communication between people is in a classroom at school; the teacher's role is characteristically to provide information, show examples, ask questions, and provide feedback on student answers. A student characteristically undertakes learning activities such as listening, asking and answering questions. However, there may be students who are deaf or blind, who have difficulty in learning or using technology, or international students who have difficulties in understanding a non-native language of instruction.

\section{2) Direct Interaction}

a) People to Objects $(\mathrm{P}-\mathrm{O})$

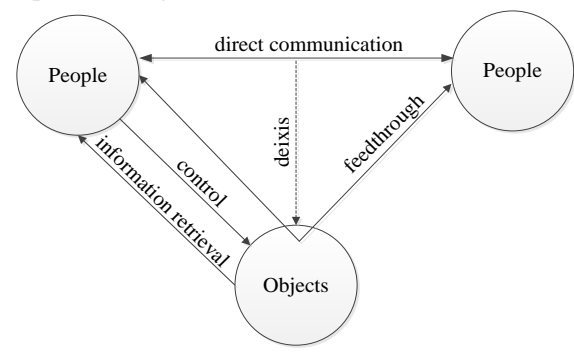

Figure 4 Interaction between people and objects

In direct interaction, people interact with objects (Fig. 4) by pointing, looking, or touching (P-O). There are two main purposes in the interaction between people and objects: control, and information retrieval. People control objects by moving, or holding them, and can receive information by looking at or touching them, perhaps while being guided by other people drawing attention to them. For example, a museum guide may point at an exhibit (deixis) when explaining its history to visitors. Blind visitors can touch the object to get information such as shape, size, and weight. In direct interaction, when a person acts upon an object (e.g. moving a piano), the other people may feel the effect of the action, which is called "feedthrough" in Dix's framework [5].

b) People to Technology $(P-T)($ Fig. 5)

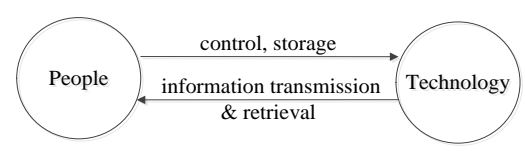

Figure 5 The interaction between people and technology

3) Technology supported communication

a) People-Technology-People (P-T-P)

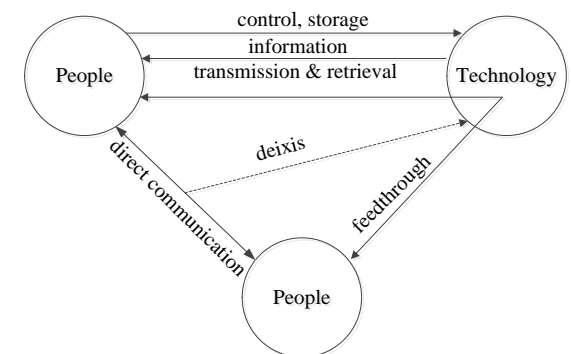

Figure 6 The interaction between people to technology to people 
Communication between people and people using technology mediation usually aims to improve that communication (Fig. 6). An example is people using their smart phones to communicate to each other by sending SMS or MMS messages, calling, sending email, sharing information through Bluetooth, or text chatting through mobile applications.

\section{b) People-Technology-Objects $(P-T-O)$}

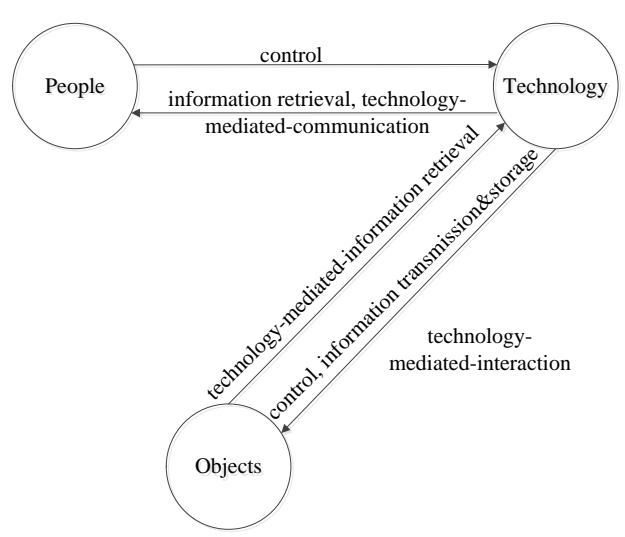

Figure 7 The interaction between people to technology to objects

The interaction between people and objects through technology mediated information retrieval (Fig. 7) is illustrated by people using (controlling) their mobile phones to take photos of a building or to scan QR codes on the building.

\section{CONCLUSION AND FUTURE WORK}

The Technology Enhanced Interaction Framework addresses the issue that, until now, there has been no framework to support technology designers and developers in considering all of the possible interactions that might occur at the same time and in the same place. Work is now in progress to provide designers with an easy to use tool that helps them apply the framework to create technology solutions to complex communication and interaction problems and situations.

\section{REFERENCES}

[1] BERNE, E. 1964. Games People Play - The Basic Hand Book of Transactional Analysis, New York, Ballantine Books.

[2] BROLL, G., SIORPAES, S., RUKZIO, E., PAOLUCCI, M., HAMARD, J., WAGNER, M. \& SCHMIDT, A. Supporting Mobile Service Usage through Physical Mobile Interaction. Pervasive Computing and Communications, 2007. PerCom '07. Fifth Annual IEEE International Conference on, 19-23 March 2007 2007. 262-271.

[3] DAVIS, K. 1977. Human Behavior At Work, New York, McGraw-Hill Book Co.

[4] DIX, A. 1995. Cooperation without (reliable) communication: Interfaces for mobile applications. Distributed Systems Engineering, 2, 171.

[5] DIX, A. 1997. Challenges for Cooperative Work on the Web: An Analytical Approach. Computer Supported Cooperative Work (CSCW), 6, 135-156.

[6] DIX, A., FINLAY, J., ABOWD, D. G. \& BEALE, R. 2004. HumanComputer Interaction, Madrid, Spain, Prentice Hall.

[7] DIX, A. J. 1994. Computer supported cooperative work - a framework. Springer Verlag, 23-37.

[8] ELLIS, J. R., RIDOLFI \& G. \& ZWIRNER, F. 1991. Groupware: Some issues and experiences. Communications of the ACM, 34

[9] FOULGER, D. 2004. Models of the Communication Process. Available: http://davis.foulger.info/research/unifiedModelOfCommunication.htm.

[10] GAINES, B. R. 1988. A conceptual framework for person-computer interaction in complex systems. Systems, Man and Cybernetics, IEEE Transactions on, 18, 532-541.

[11] HSI, S. \& FAIT, H. 2005. RFID enhances visitors' museum experience at the Exploratorium. Commun. ACM, 48, 60-65.

[12] KLINK, M. 2006. The use of interaction methods in a blended learning environment. master, University of Twente.

[13] LARSON, A. J., RAMAN, T. V. \& RAGGETT, D. 2003. W3C Multimodal Interaction Framework. Available: http://www.w3.org/TR/mmi-framework/.

[14] LAURILLARD, D. 1993. Rethinking University Teaching: a framework for the effective use of educational technology, London, Routledge.

[15] LEE, D. S., ARMitAGE, S., GROVES, P. \& STEPHENS, C. 2009. Systems for supporting group learning

[16] MERRILL, M. D. (2008). Reflections on a four decade search for effective, efficient and engaging instruction. In M. W. Allen (Ed.), Michael Allen's 2008 e-Learning Annual (Vol. 1, pp. 141-167): Wiley Pfieffer.

[17] RUKZIO, E. A. B., GREGOR AND WETZSTEIN, SERGEJ 2008. The Physical Mobile Interaction Framework (PMIF). Technical Report LMU-MI-2008-2.

[18] SILVERMAN, D. 1998. Harvey Sacks Social Science \& Conversation Analysis, New York, Oxford University Press.

[19] SUNG, Y.-T., CHANG, K.-E., HOU, H.-T. \& CHEN, P.-F. 2010. Designing an electronic guidebook for learning engagement in a museum of history. Computers in Human Behavior, 26, 74-83.

[20] VYAS, D., DIX, A. \& NIJHOLT, A. 2008. Role of Artefacts in Mediated Communication. CHI 2008. Florence, Italy: ACM SIGCHI.

[21] WANG, S.-K. 2008. The Effects of a Synchronous Communication Tool (Yahoo Messenger) on Online Lerners' Sense of Community and their Multimedia Authoring Skills. Journal of Interactive Online Learning, 7. 